\title{
Circuit Models of Lossy Multiconductor Transmission Lines: Incident Plane Wave Effect
}

\author{
Saih Mohamed ${ }^{1}$, Rouijaa Hicham², Ghammaz Abdelilah ${ }^{1}$ \\ ${ }^{1}$ Laboratory of Electrical Systems and Telecommunications, Department of Physics, Faculty of Sciences and \\ Technology, Cadi Ayyad university, Marrakesh, Morocco. \\ ${ }^{2}$ Laboratory for Systems Analysis and Information Processing, Department of Applied Physics, Faculty of Sciences and \\ Technology, Hassan I university, Settat, Morocco
}

\begin{tabular}{|c|c|}
\hline Article Info & ABSTRACT \\
\hline $\begin{array}{l}\text { Keyword: } \\
\text { Branin's method } \\
\text { ESACAP } \\
\text { Incident plane wave } \\
\text { Multiconductor transmission } \\
\text { lines }\end{array}$ & $\begin{array}{l}\text { In this paper, we concentrate on the variety impacts of incident plane wave } \\
\text { on multiconductor transmission lines, utilizing Branin's method, which is } \\
\text { alluded to as the method of characteristics. The model can be directly used } \\
\text { for the time-domain and frequency-domain analyses, Moreover, it had the } \\
\text { advantage of being used without the need of setting the preconditions of the } \\
\text { charges applied to its ends; this permits it to be effortlessly embedded in } \\
\text { circuit simulators, for example Spice, Saber, and Esacap. This model validity } \\
\text { is affirmed by contrasting our simulation results under ESACAP and } \\
\text { different results, and we will talk about variety impacts of incident plane } \\
\text { wave. }\end{array}$ \\
\hline \multicolumn{2}{|l|}{ Corresponding Author: } \\
\hline \multicolumn{2}{|c|}{$\begin{array}{l}\text { Saih Mohamed, } \\
\text { Laboratory of Electrical Systems and Telecommunications, Department of Physics, } \\
\text { Faculty of Sciences and Technology, Cadi Ayyad university, } \\
\text { Marrakesh, Morocco. } \\
\text { Email: saih.mohamed@gmail.com }\end{array}$} \\
\hline
\end{tabular}

\section{INTRODUCTION}

A significant percentage of electric and electronic systems communicate through interconnecting wiring harnesses that can be vulnerable to external electromagnetic (EM) interference. Consequently, it is thusly profoundly vital for Electromagnetic Compatibility (EMC) studies to develop software tools capable of predicting induced effects in cables configurations [1],[2].

The forecast of these disturbances, which are typically impelled by outside fields or lumped sources, is an established issue which can be managed in an assortment of ways. Henceforth, it can be dealt with in the frequency domain, and therefore the induced responses of cables are fathomed by utilizing Multiconductor transmission line (MTL) theory [3],[4].

In a pioneering work, Taylor [5] exploited the transmission line (TL) theory to predict the response of a TL excited by an external electromagnetic field. The case studied was a two-wire line system in free space excited by a plane wave field. Since then, many other authors contributed to the refinement of the method. In particular, Paul [6] extended the work of Taylor to the case of (MTLs) and later, Agrawal [7] formulated field-to-wire coupling in terms of the electric field only.

As of late, a considerable measure of researches have been done on the advancement of simulation program with integrated circuit emphasis (SPICE) equal circuit models for MTL energized by an episode electromagnetic field [8]. In any case, the proposed models depend on one supposition that the TLs are lossless. After then, some lossless models have been proposed to consider the variety impacts of the occurrence plane wave on a MTL [9].These models can be implemented to compute transient responses 
without the inverse Fourier Transform (IFT), and directly connected to nonlinear and time-varying terminators with the models already available in SPICE.

In this paper, an equivalent circuit model for the analyses of the radiated susceptibility of lossy MTL, the model is substantial in the time and frequency domain with linear and nonlinear loads and effortlessly brought into the circuit simulators, such as Spice and ESACAP [10],[11]. The variation effect of incident plane wave on coaxial cable is studied with the proposed model. The method is validated by comparing results with other methods.

\section{DESCRIPTION OF MTL}

\subsection{Model of MTL}

The Telegrapher's equations for a MTL in the presence of external electromagnetic radiation, such as those radiated by the plane wave are written as

$$
\left\{\begin{array}{l}
\frac{\partial V(z, t)}{\partial z}+R I(z, t)+L \frac{\partial I(z, t)}{\partial t}=V_{f}(z, t) \\
\frac{\partial I}{\partial z}+G V(z, t)+C \frac{\partial V}{\partial t}=I_{f}(z, t)
\end{array}\right.
$$

Where $V(z, t)=\left[V_{1}(z, t), V_{2}(z, t), \ldots, V_{N}(z, t)\right]^{T}$ and $I(z, t)=\left[I_{1}(z, t), I_{2}(z, t), \ldots, I_{N}(z, t)\right]^{T}$ represent the voltage (with respect to the ground) and current vectors of the line, and $L, C, R$ and $G$ are the per-unitlenght (p.u.l) inductance, capacitance, resistance, and conductance matrices of the line, respectively.

The $n \times 1$ vectors, $V_{f}(z, t)$ and $I_{f}(z, t)$, are distributed sources that represent external excitation of the transmission line and are resolved utilizing a proper coupling model. There are a few alternatively equivalent, usually utilized coupling models created by Taylor et al. [5], Agrawal et al. [7], and Rachidi [12]. In this paper, we are utilizing the extended version of the formulation developed by Taylor, Satterwhite, and Harrison. In this model, the dispersed excitation sources are depicted as far as the vertical and horizontal component of the incident electric field. For MTL, as appeared in Figure 1. We have:

$$
\begin{aligned}
& V_{f}=\left[E_{z}^{i n c}(h, z, t)-E_{z}^{i n c}(0, z, t)\right]-\frac{\partial}{\partial z} \int_{0}^{h} E_{x}^{i n c}(x, z, t) d x \\
& I_{f}=-C \frac{\partial}{\partial t} \int_{0}^{h} E_{x}^{i n c}(x, z, t) d x
\end{aligned}
$$

Where $\mathrm{h}$ is the height of the line, and $E_{z}^{i n c}(h, z, t)$ and $E_{x}^{i n c}(x, z, t)$ are the horizontal and vertical components of the incident electric field, respectively. The incident field, in the absence of the line, as shown in Figure 2, can be written in the following frequency form

$$
\vec{E}^{i n c}(x, y, z, \omega)=E_{0}\left(e_{x} \overrightarrow{a_{x}}+e_{y} \overrightarrow{a_{y}}+e_{z} \overrightarrow{a_{z}}\right) e^{-j \beta_{x} x} e^{-j \beta_{y} y} e^{-j \beta_{z} z}
$$




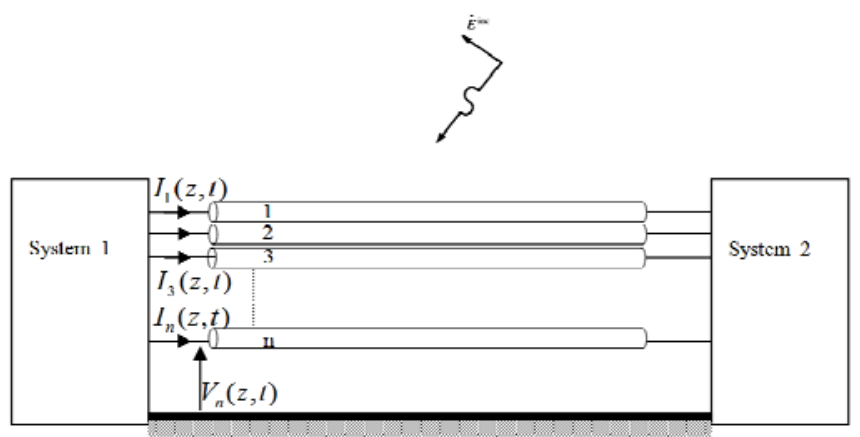

Figure 1. A Multiconductor transmission line over an infinite and perfectly conducting ground
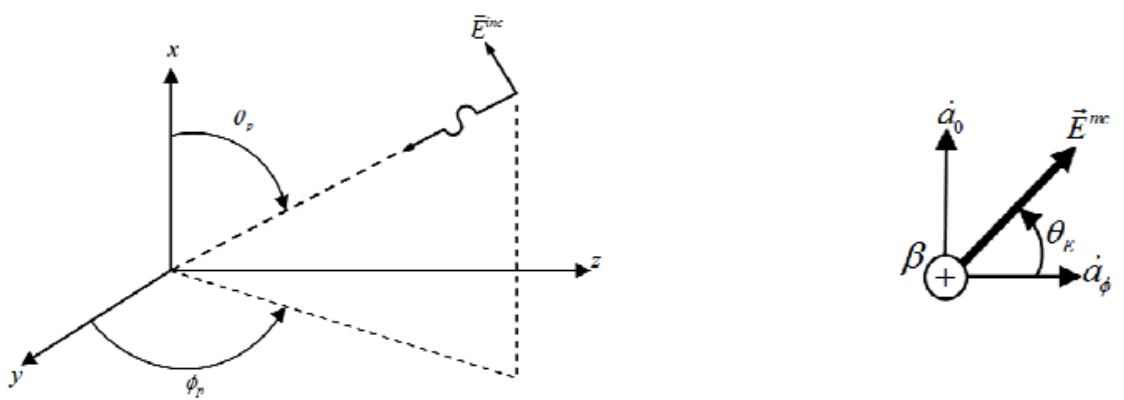

Figure 2. Definitions of the parameters characterizing the incident field as a uniform plane wave

Where $e_{x}, e_{y}$ and $e_{z}$ are three unit vectors in the Cartesian coordinate system given by:

$$
\left\{\begin{array}{l}
e_{x}=\sin \theta_{E} \sin \theta_{P} \\
e_{y}=-\sin \theta_{E} \cos \theta_{P} \cos \phi_{P}-\cos \theta_{E} \sin \phi_{P} \\
e_{z}=-\sin \theta_{E} \cos \theta_{P} \sin \phi_{P}+\cos \theta_{E} \cos \phi_{P} \\
e_{x}+e_{y}+e_{z}=1
\end{array}\right.
$$

The angle $\theta_{E}$ defines the polarization type. The polarization is horizontal if $\theta_{E}$ is equal to zero and vertical if it is equal to $90^{\circ}$. The angle $\theta_{p}$ determines the elevation relative to the ground. This angle is commonly called the incident angle. The angle $\phi_{p}$ gives the propagation direction relative to the axis $\mathrm{Oz}$. The components of the phase constant along those coordinate axes are:

$$
\left\{\begin{array}{l}
\beta_{x}=-\beta \cos \theta_{P} \\
\beta_{y}=-\beta \sin \theta_{P} \cos \phi_{P} \\
\beta_{z}=-\beta \sin \theta_{P} \sin \phi_{P}
\end{array}\right.
$$

The phase constant is related to the frequency and properties of the medium as:

$$
\beta=\omega \sqrt{\mu \varepsilon}=\frac{1}{v_{0}} \sqrt{\mu_{r} \varepsilon_{r}}
$$

Where $v_{0}=\frac{1}{\sqrt{\mu_{0} \varepsilon_{0}}}$ is the phase velocity in the space and the medium is characterized by the permeability $\mu=\mu_{0} \mu_{r}$ and permittivity $\varepsilon=\varepsilon_{0} \varepsilon_{r}$. 
For the situation where the line is situated over a ground plane, as appeared in Figure 3, the connected field turns into the total of the incident field and the ground-reflected field

$$
\vec{E}_{\text {totale }}^{\text {inc }}=\vec{E}^{\text {inc }}+\vec{E}^{\text {ref }}
$$

These field components are as follows:

$$
\begin{aligned}
& \vec{E}^{i n c}(x, y, z, \omega)=E_{0}\left(e_{x} \overrightarrow{a_{x}}+e_{y} \overrightarrow{a_{y}}+e_{z} \overrightarrow{a_{z}}\right) e^{-j \beta_{x} x} e^{-j \beta_{y} y} e^{-j \beta_{z} z} \\
& \vec{E}^{r e f}(x, y, z, \omega)=E_{0}\left(e_{x} \overrightarrow{a_{x}}-e_{y} \overrightarrow{a_{y}}-e_{z} \overrightarrow{a_{z}}\right) e^{j \beta_{x} x} e^{-j \beta_{y} y} e^{-j \beta_{z} z}
\end{aligned}
$$

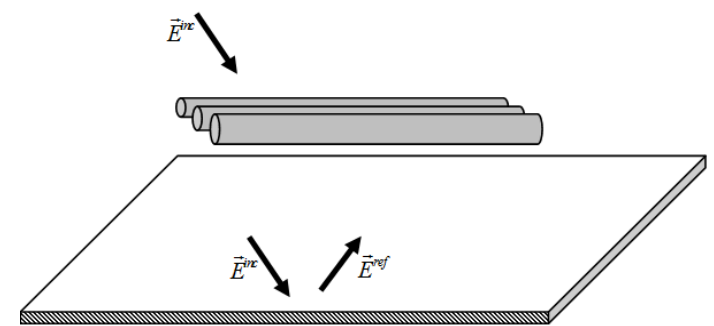

Figure 3. Configuration with the presence of a perfectly conducting ground plane

The total field is defined by:

$$
\begin{aligned}
& \vec{E}_{\text {totale }}^{\text {inc }}=\vec{E}^{\text {inc }}+\vec{E}^{\text {ref }}=\vec{E}_{\text {totale }_{x}}^{\text {inc }} \vec{a}_{x}+\vec{E}_{\text {totale }_{y}}^{\text {inc }} a_{y}+\vec{E}_{\text {totale }_{z}}^{\text {inc }} \vec{a}_{z} \\
& \vec{E}_{\text {totale }_{x}}^{\text {inc }}=2 E_{0} e_{x} \cos \left(\beta_{x} x\right) e^{-j \beta_{y} y} e^{-j \beta_{z} z} \\
& \vec{E}_{\text {totale }_{y}}^{\text {inc }}=-2 j E_{0} e_{y} \sin \left(\beta_{x} x\right) e^{-j \beta_{y} y} e^{-j \beta_{z} z} \\
& \vec{E}_{\text {totale }_{z}}^{\text {inc }}=-2 j E_{0} e_{z} \sin \left(\beta_{x} x\right) e^{-j \beta_{y} y} e^{-j \beta_{z} z}
\end{aligned}
$$

\subsection{Equivalent Circuit Model for Lossy MTL}

The equations in (1) are coupled sets of partial differential equations. To decouple them a similar transformation is required [3],[13]. Characterizing the change to mode amounts as

$$
\left\{\begin{array}{l}
V=T_{V} V_{m} \\
I=T_{I} I_{m}
\end{array}\right.
$$

Substituting (15) into (1) gives, the system of equations for the inner conductors becomes

$$
\left\{\begin{array}{l}
\frac{\partial}{\partial z} V_{m}+R_{m} I_{m}(z, t)+L_{m} \frac{\partial I_{m}(z, t)}{\partial t}=V_{f m}(z, t) \\
\frac{\partial}{\partial z} I_{m}+G_{m} V_{m}(z, t)+C_{m} \frac{\partial V_{m}(z, t)}{\partial t}=I_{f m}(z, t)
\end{array}\right.
$$


Where

$$
\begin{aligned}
& L_{m}=T_{V}^{-1} L T_{I} \\
& C_{m}=T_{I}^{-1} C T_{V} \\
& R_{m}=T_{V}^{-1} R T_{I} \\
& G_{m}=T_{I}^{-1} G T_{V} \\
& V_{f m}=T_{V}^{-1} V_{f} \\
& I_{f m}=T_{I}^{-1} I_{f}
\end{aligned}
$$

Both $L_{m}$ and $C_{m}$ are diagonal matrices of dimension $N x N, T_{V}$ and $T_{I}$ are selected so that the matrices $L_{m}$ and $C_{m}$ are diagonals. After calculating $L_{m}, C_{m}$ and $R_{m}$ matrices, we calculate the mode characteristic impedance, utilizing the primary term of the Taylor series expansion, we get

$$
Z_{c m_{i i}}=\sqrt{\frac{R_{m_{i i}} L_{m_{i i}}}{C_{m_{i i}}}} \approx R_{c m_{i}}+\frac{1}{j C_{m f_{i}} \omega}, \quad \mathrm{R}_{m_{i i}}<<L_{m_{i i}} \omega \quad i=1 \ldots N
$$

Where

$$
R_{c m_{i}}=\sqrt{\frac{L_{m_{i i}}}{C_{m_{i i}}}}
$$

The characteristic impedance in this case, is presented as a characteristic resistance $R_{c m_{i}}$ and capacity $C_{m f_{i}}=\frac{2 L_{m_{i i}}}{R_{m_{i i}} R_{c m_{i}}}$, as shown in Figure 4. With the same approximation, the constant of propagation becomes:

$$
\gamma_{m_{i}}=\alpha_{m_{i}}+j \beta_{m_{i}}=\frac{R_{m_{i i}}}{2 R_{c m_{i}}}+j \omega \sqrt{L_{m_{i i}} C_{m_{i i}}}
$$

The solution of (16) in the frequency domain can be found in [11],[14]. And is given by

$$
\left\{\begin{array}{l}
V_{m}(L)=\varphi_{m 11}(L) V_{m}(0)+\varphi_{m 12}(0) I_{m}(0)+V_{f t m}(L) \\
I_{m}(L)=\varphi_{m 21}(L) V_{m}(0)+\varphi_{m 22}(0) I_{m}(0)+I_{f t m}(L)
\end{array}\right.
$$

Where the modal chain-parameter submatrices become

$$
\begin{aligned}
& \varphi_{m 11}(\Delta z)=\frac{1}{2}\left(e^{\gamma_{m} L}+e^{-\gamma_{m} L}\right) \\
& \varphi_{m 12}(\Delta z)=-\frac{1}{2} Z_{c m}\left(e^{\gamma_{m} L}-e^{-\gamma_{m} L}\right)
\end{aligned}
$$




$$
\begin{aligned}
& \varphi_{m 21}(\Delta z)=-\frac{1}{2} Z_{c m}^{-1}\left(e^{\gamma_{m} L}-e^{-\gamma_{m} L}\right) \\
& \varphi_{m 22}(\Delta z)=\frac{1}{2}\left(e^{\gamma_{m} L}+e^{\gamma_{m} L}\right)
\end{aligned}
$$

$V_{f t m}(L)$ and $I_{f t m}(L)$ are the total modal forcing functions due to the incident field, and are given by

$$
\begin{aligned}
& V_{f t m}(L)=\int_{0}^{L}\left[\varphi_{m 11}(L-\tau) V_{f m}(\tau)+\varphi_{m 12}(L-\tau) I_{f m}(\tau)\right] d \tau \\
& I_{f t m}(L)=\int_{0}^{L}\left[\varphi_{m 21}(L-\tau) V_{f m}(\tau)+\varphi_{m 22}(L-\tau) I_{f m}(\tau)\right] d \tau
\end{aligned}
$$

Substituting (22) into (21) gives

$$
\left\{\begin{array}{l}
V_{m}(0)-Z_{c m} I_{m}(0)=e^{-\gamma_{m} L}\left[V_{m}(L)-Z_{c m} I_{m}(L)\right]+E_{0}(L) \\
V_{m}(L)+Z_{c m} I_{m}(L)=e^{-\gamma_{m} L}\left[V_{m}(0)+Z_{c m} I_{m}(0)\right]+E_{L}(L)
\end{array}\right.
$$

Where

$$
\left\{\begin{array}{l}
E_{0}(L)=-e^{-\gamma_{m} L}\left[V_{f t m}(L)-Z_{c m} I_{f t m}(L)\right] \\
E_{L}(L)=\left[V_{f t m}(L)+Z_{c m} I_{f t m}(L)\right]
\end{array}\right.
$$

Recognizing the basic time-delay transformation:

$$
e^{ \pm j \omega T} F(\omega)=\boldsymbol{F}(t-T)
$$

These become, in the time domain,

$$
\left\{\begin{array}{l}
V_{m}(0, t)-Z_{c m} I_{m}(0, t)=e^{-\alpha_{m} L}\left[V_{m}\left(L, t-T_{m}\right)-Z_{c m} I_{w}\left(L, t-T_{m}\right)\right]+E_{0}(t) \\
V_{m}(L, t)+Z_{c m} I_{m}(L, t)=e^{-\alpha_{m} L}\left[V_{m}\left(0, t-T_{m}\right)+Z_{c m} I_{m}\left(0, t-T_{m}\right)\right]+E_{L}(t)
\end{array}\right.
$$

Were $T_{m}$ is the one-way delay of the wires, and is denoed by $T_{m}=L \sqrt{L_{m} C_{m}}$. The additional sources are

$$
\left\{\begin{array}{l}
E_{0}(t)=-e^{-\alpha_{m} L}\left[V_{f t m}\left(L, t-T_{m}\right)-Z_{c m} I_{f t m}\left(L, t-T_{m}\right)\right] \\
E_{L}(t)=\left[V_{f t m}(L, t)+Z_{c m} I_{f t m}(L, t)\right]
\end{array}\right.
$$

The terms of the 'controlled' generators of voltage and current placed in each conductor of the cell are:

$$
\begin{aligned}
& V_{i}(z, t)=\sum_{k=1}^{N}\left\{\left[T_{V}\right]_{i k} V_{m_{k}}(z, t)\right\} \\
& I_{m_{i}}(z, t)=\sum_{k=1}^{N}\left\{\left[T_{I}\right]_{i k}^{-1} I_{k}(z, t)\right\}
\end{aligned}
$$




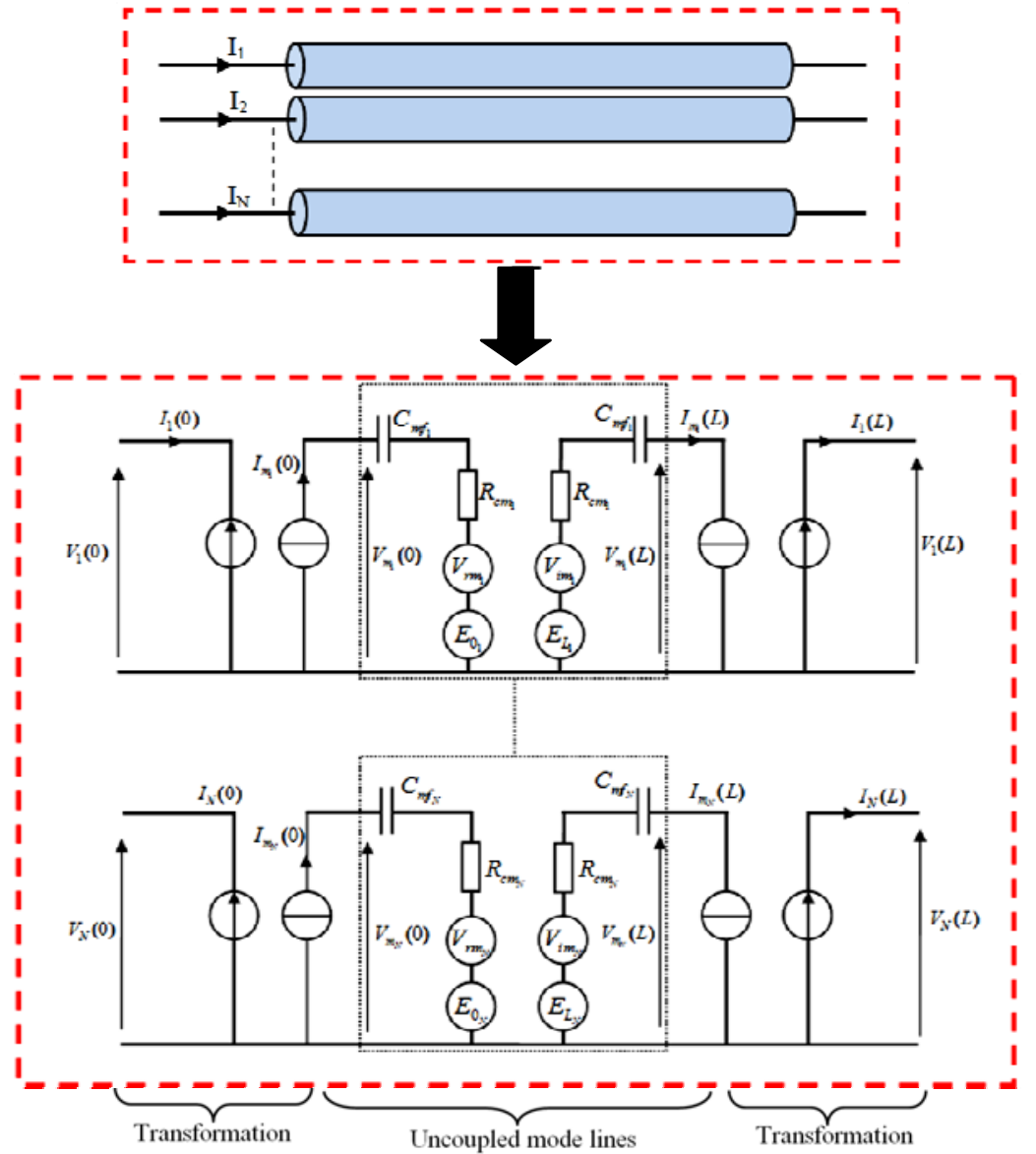

Figure 4. Circuit model of lossy multiconductor transmission line

\section{SIMULATION RESULTS AND VALIDATION}

\subsection{Radiated Susceptibility Analysis of Three conductors}

The analysis of the radiated immunity is carried out on three conductors excited by an incident plane wave as shown in Figure 5. The length $\mathrm{L}$ and the radius $\mathrm{r}$ of the lines are $0.2 \mathrm{~mm}$ and $2 \mathrm{~m}$, respectively. The distance $\mathrm{d}$ between the two wires is $1.27 \mathrm{~mm}$, the dielectric thicknesses $\mathrm{t}$ is $0.25 \mathrm{~mm}$, and the dielectric constant $\varepsilon_{\mathrm{r}}$ is 3.5 . The loads $\mathrm{R}_{1}, \mathrm{R}_{2}, \mathrm{R}_{3}$, and $\mathrm{R}_{4}$ of the lines are $500 \Omega$. The external field, oriented along $\mathrm{x}$ and propagating along $\mathrm{z}$ axis $\left(\mathrm{E}_{\mathrm{x}}-\mathrm{K}_{\mathrm{z}}\right)$. The variation of the electric field is defined by a ramp rise time $t_{\mathrm{r}}=1 \mathrm{~ns}$ and the amplitude $\mathrm{E}_{0}=1 \mathrm{~V} / \mathrm{m}$, as shown in Figure 6.
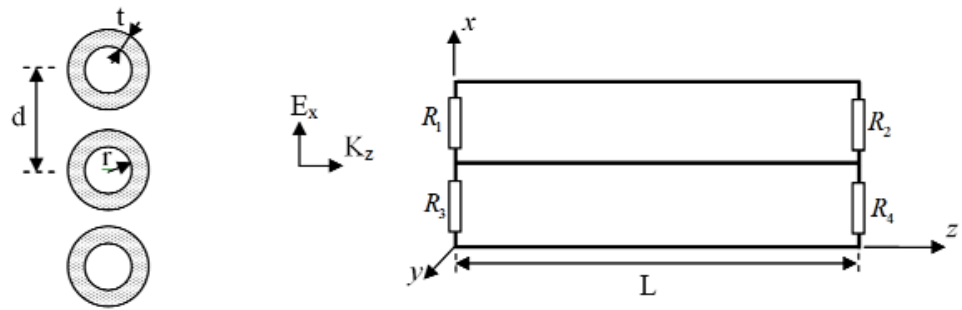

Figure 5. (a) Geometrical cross-section of wires. (b) Configuration of the simulation for radiated analysis 


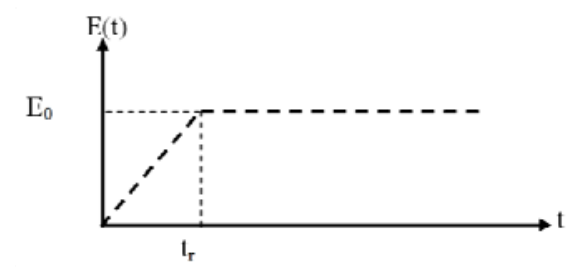

Figure 6. Electric field variation is defined by ramp rise time $\mathrm{t}^{\mathrm{r}}=1 \mathrm{~ns}$

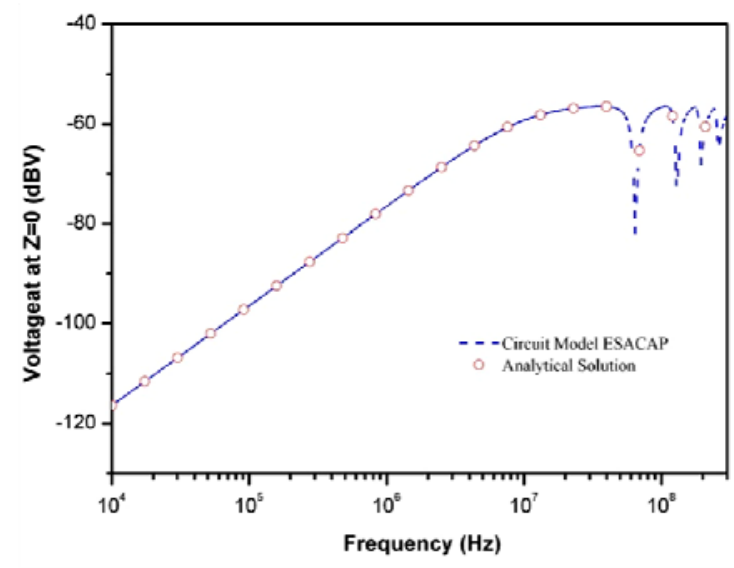

Figure 7a. Voltage responses at the near-end in the frequency analysis when the incident wave propagates along the $\mathrm{z}$-axis obtained by different methods

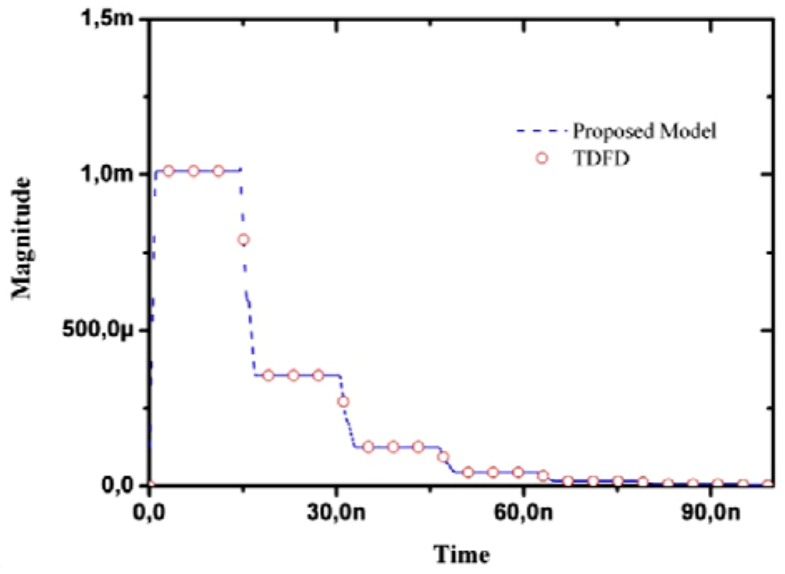

Figure $7 \mathrm{~b}$. Voltage responses at the near-end in the transient analysis when the incident wave propagates along the $\mathrm{z}$-axis obtained by different methods

The near-end voltage of the line obtained by the proposed model is shown in Figure $7 \mathrm{~b}$ together with the result derived by the FDTD [1], where the "FDTD" implies the finite difference time domain solution to the transmission-line equations of the cable. The different solutions are in a very good agreement.

Figure $7 \mathrm{a}$ demonstrates the magnitude of the frequency responses of the near-end voltage acquired by the proposed model. The outcomes acquired by the ESACAP test system are in great concurrence with the analytical solution [13].

\subsection{Radiated Susceptibility Analysis of Three conductors}

The configuration used for the radiated susceptibility analysis is shown in Figure 8. The height $\mathrm{h}$ and the length $\mathrm{L}$ are $2 \mathrm{~cm}$ and $1 \mathrm{~m}$, respectively. The wire radius $\mathrm{r}$ is $0.25 \mathrm{~mm}$, and the relative permittivity $\varepsilon_{r}$ is 2.25. the line is terminated with short circuit at the far-end $\left(Z_{b}=0.5 \Omega\right)$. The per-unit-length dc resistance of the wire is $r_{d c}=1.3 \Omega / m$. The normalized incident field $E=1 \mathrm{~V} / \mathrm{m}$.

The Analysis performed for three reference field directions as described in Figure 8 are as follow:

a) Ex, $\mathrm{Kz}$ is the vertical electric field (parallel to terminations) and propagation vector along the line.

b) Ex, Ky is the vertical electric field and propagation vector horizontal and orthogonal to the line

c) $\mathrm{Ez}, \mathrm{Kx}$ is the horizontal electric field ( parallel to line) and propagation vector vertical to GND-plane

Figure 9 demonstrates that the voltage reaction at the cable ends in the frequency analysis with the incident wave. For all cases, when the line is ended with short circuit at the far-end and open circuit at the near-end $\left(Z_{1}=5.10^{8} \Omega\right.$ and $\left.Z_{2}=0.5 \Omega\right)$, the line resonates at $f=n \times\left(3.10^{8} 4 / \lambda\right), n=1,3,5 \ldots\left(f_{1}=75 \mathrm{MHz}\right.$, $\left.\mathrm{f}_{2}=225 \mathrm{MHz}, \mathrm{f}_{3}=375 \mathrm{MHz} \ldots\right)$. The configuration with both sides associated with the ground $\left(\mathrm{Z}_{1}=\mathrm{Z}_{2}=0.5 \Omega\right)$, for cases (a) and (c), eliminates practically all resonance, and the immunity is enhanced more than $50 \mathrm{~dB}$. For case (b), the resonances are located $a_{f}=n \times\left(3.10^{8} 2 / \lambda\right), n=1,3,5 \ldots\left(f_{1}=150 M H z, f_{2}=450 M H z \ldots\right)$. The simulations have affirmed that the perfect configuration requests the establishing of the line at both sides. 


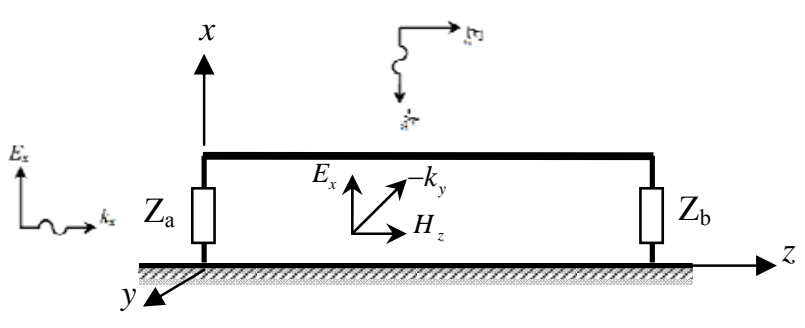

Figure 8. Single line over an infinite and perfectly conducting ground excited by an incident plane

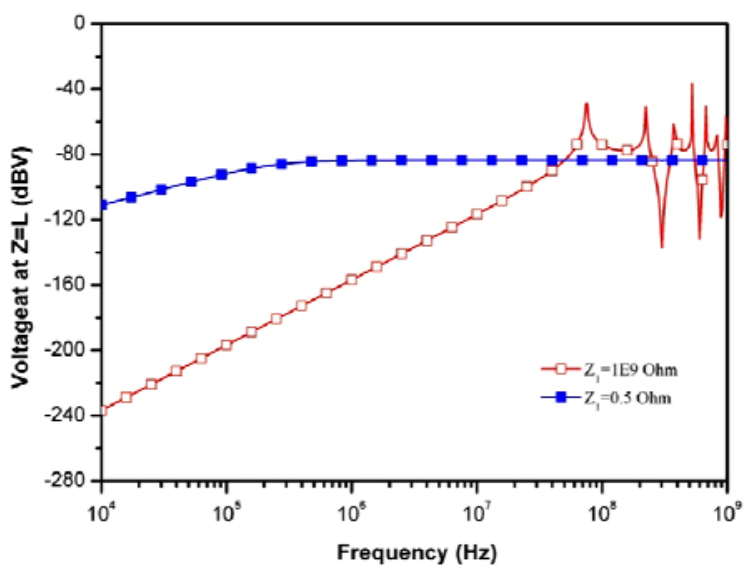

Figure 9c. Case 3- Voltage responses at the cable ends in the frequency analysis with the incident wave with $\phi_{p}=0^{\circ}, \theta_{p}=0^{\circ}$ and $\theta_{E}=0^{\circ}$

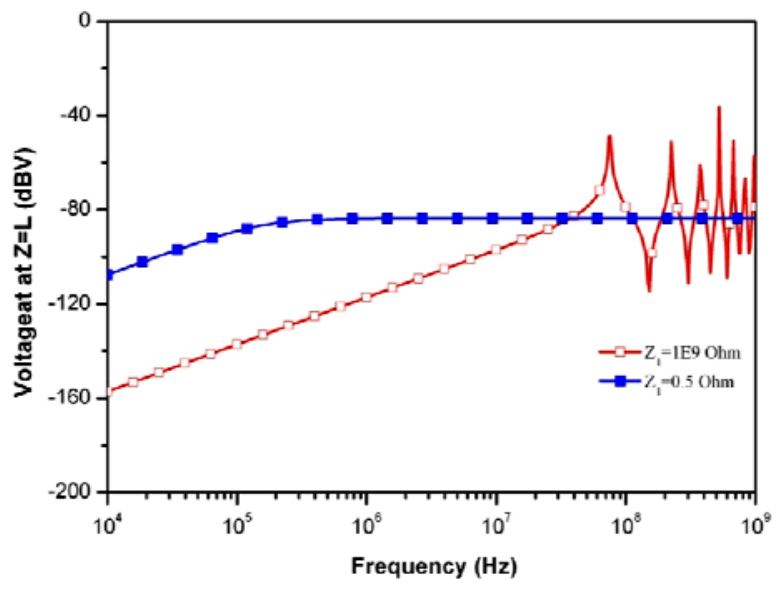

Figure 9a. Case 1- Voltage responses at the cable ends in the frequency analysis with the incident wave with $\phi_{p}=-90^{\circ}, \theta_{p}=90^{\circ}$ and $\theta_{E}=90^{\circ}$

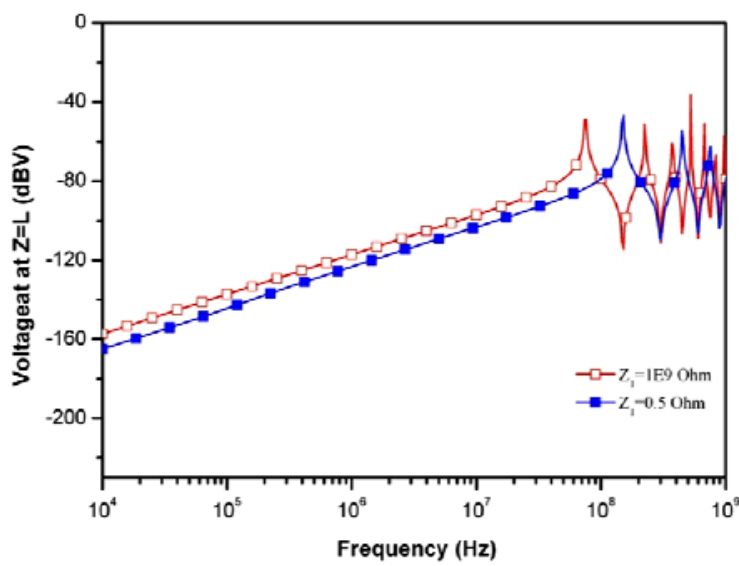

Figure 9b. Case 2- Voltage responses at the cable ends in the frequency analysis with the incident wave with $\phi_{p}=0^{\circ}, \theta_{p}=90^{\circ}$ and $\theta_{E}=90^{\circ}$

\section{CONCLUSION}

Circuit models for the examination of the radiated and conducted susceptibilities for lossy MTL have been exhibited. The principle point of interest of these models comprises in the likelihood of utilizing them as a part of frequency and time domains, with linear and non linear loads individually. A detailed description of MTL has been presented. The legitimacy performed by contrasting the circuit test system results and the arrangements inferred by alternate techniques has uncovered an agreeable precision.

For the variation effects of the incident angle on a MTL line, a model of a transmission line referenced to a ground plane excited by an external plane wave is studied. We can find that the best arrangement requests the grounding of the line at both sides. It is easy to extend the models to Multiconductor shielded cables excited by a uniform and non-uniform incident wave. This inquiry will be talked about in a further study.

\section{REFERENCES}

[1] N. Ghaffarzadeh, "A New Method for Recognition of Arcing Faults in Transmission Lines using Wavelet Transform and Correlation Coefficient," Indones. J. Electr. Eng. Inform. IJEEI, vol/issue: 1(1), 2013.

[2] A. Narwan, et al., "Backpropagation Neural Network Modeling for Fault Location in Transmission Line $150 \mathrm{kV}$," Indones. J. Electr. Eng. Inform. IJEEI, vol/issue: 2(1), 2014.

[3] C. R. Paul, "Analysis of multiconductor transmission lines," New York, Wiley, 1994. 
[4] C. R. Paul, "Efficient Numerical Computation of the Frequency Response of Cables Illuminated by an Electromagnetic Field (Short Papers)," IEEE Trans. Microw. Theory Tech., vol/issue: 22(4), pp. 454-457, 1974.

[5] C. Taylor, et al., "The response of a terminated two-wire transmission line excited by a nonuniform electromagnetic field," IEEE Trans. Antennas Propag., vol/issue: 13(6), pp. 987-989, 1965.

[6] C. Paul, "Frequency Response of Multiconductor Transmission Lines Illuminated by an Electromagnetic Field," IEEE Trans. Electromagn. Compat, vol/issue: EMC-18(4), pp. 183-190, 1976.

[7] A. Agrawal, et al., "Transient response of multiconductor transmission lines excited by a nonuniform electromagnetic field," vol. 18, pp. 432-435, 1980.

[8] H. Xie, et al., "A Hybrid FDTD-SPICE Method for Transmission Lines Excited by a Nonuniform Incident Wave," IEEE Trans. Electromagn. Compat, vol/issue: 51(3), pp. 811-817, 2009.

[9] Y. Mejdoub, et al., "Variation effect of plane-wave incidence on multiconductor transmission lines," Int. J. Microw. Wirel. Technol., pp. 1-8, 2015.

[10] Y. Mejdoub, et al., "Optimization circuit model of a multiconductor transmission line," Int. J. Microw. Wirel. Technol., vol/issue: 6(06), pp. 603-609, 2014

[11] M. Saih, et al., "Circuit Models for Conducted Susceptibility Analyses of Multiconductor Shielded Cables," Int. J. Electr. Comput. Energ. Electron. Commun. Eng., vol/issue: 9(4), 2015.

[12] F. Rachidi, "Formulation of the field-to-transmission line coupling equations in terms of magnetic excitation field," IEEE Trans. Electromagn. Compat, vol/issue: 35(3), pp. 404-407, 1993.

[13] M. Saih, et al., "Circuit models of multiconductor shielded cables: incident plane wave effect," Int. J. Numer. Model. Electron. Netw. Devices Fields, vol/issue: 29(2), pp. 243-254, 2016.

[14] S. Caniggia and F. Maradei, "SPICE-Like Models for the Analysis of the Conducted and Radiated Immunity of Shielded Cables," IEEE Trans. Electromagn. Compat, vol/issue: 46(4), pp. 606-616, 2004.

\section{BIOGRAPHIES OF AUTHORS}

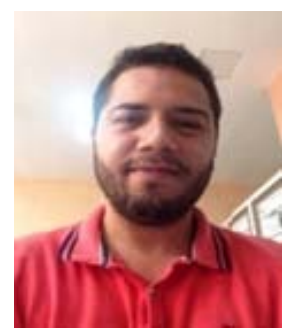

Mohamed SAIH received the Engineer Diploma in Electrical Systems and Telecommunications in 2011 from Cadi Ayyad University, Marrakech Morocco, where he is currently working toward the Ph.D degree at the Department of Applied Physics, Electrical Systems and Telecommunications Laboratory, Cadi Ayyad University of Marrakech, Morocco. His research interests include electromagnetic compatibility, multiconductor transmission lines, numerical electromagnetic methods and antenna designs.

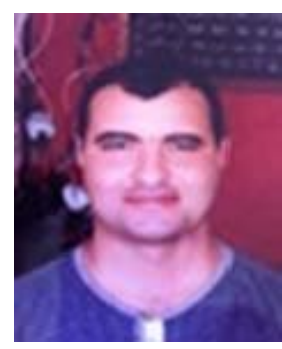

Hicham ROUIJAA is a Professor of physics, attached to Cadi Ayyad University, Marrakesh Morocco. He is obtained his PhD Thesis on \" Modeling of Multiconductor Transmission Lines using Pade approximant method: Circuit model \", in 2004, from Aix Marseille University France. He is associate member of Electrical Systems and Telecommunications Laboratory LSET at the Cadi Ayyad University. His current research interests concern electromagnetic compatibility and multiconductor transmission lines.

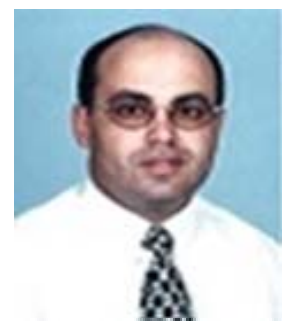

Abdelilah GHAMMAZ received the Doctor of Electronic degree from the National polytechnic Institut (ENSEEIHT) of Toulouse, France, in 1993. In 1994 he went back to Cadi Ayyad University of Marrakech - Morroco. Since 2003, he has been a Professor at the Faculty of Sciences and technology, Marrakech, Morroco. He is a member of ELectrical Systems and Telecommunications Laboratory LSET at the Cadi Ayyad University. His research interests in the field of electromagnetic compatibility, multiconductor transmission lines. 